\title{
Differential Expression of IncRNA CASC2 in the Serum of Childhood Asthma and Its Role in Airway Smooth Muscle Cells Proliferation and Migration
}

\author{
Yane Yang', Zhihong Sun', Tingting Ren', Wei Lei ${ }^{2}$ \\ 'Department of Pediatrics, The Second Affiliated Hospital of Xi'an Medical University, Xi'an, Shaanxi, People's Republic of China; ${ }^{2}$ Department of \\ Pediatrics, Chang 'an District Hospital, Xi'an, Shaanxi, People's Republic of China \\ Correspondence: Zhihong Sun, Department of Pediatrics, The Second Affiliated Hospital of Xi'an Medical University, I67 Fang Dong Street, Xi'an, \\ 710038, People's Republic of China, Tel/Fax +86-2983553606, Email srrdge766@I63.com
}

Purpose: Asthma is a common chronic disease in children. Abnormal expression of lncRNAs can be used as biomarkers for early diagnosis of asthma. The present study aimed to explore the expression change and clinical value of lncRNA CASC2 in asthma, and further investigate its potential mechanism.

Patients and Methods: Seventy asthma children and 66 healthy controls were recruited. Levels of mRNAs were detected using qRT-PCR. Receiver operating characteristic (ROC) curves were drawn for diagnostic value evaluation. Asthma cell models were established using PDGF-BB in Human airway smooth muscle cells (ASMCs). Levels of Th1/Th2 related cytokines were detected using ELISA. Lipofectamine 3000 was used for cell transfection. The target relationship was verified using luciferase activity assay.

Results: CASC2 was at a low level in asthma children in comparison with the healthy controls. Serum CASC2 can distinguish healthy individuals from asthma children. Overexpression of CASC2 inhibited PDGF-BB induced cell proliferation and migration. CASC2 upregulation inhibited the release of Th2 related cytokines (IL-4 and IL-10), but promoted the release of Th1-related cytokine (IFN- $\gamma$ ). In PDGF-BB treated ASMCs, the reduced expression of contractile phenotype marker ( $\alpha-\mathrm{SMA})$ was detected, but the trend was reversed by CASC2 upregulation. LncRNA CASC2 serves as a ceRNA of miR-31-5p, overexpression of miR-31-5p reversed the influence of CASC2 on asthma in vitro.

Conclusion: Serum CASC2 can distinguish healthy individuals from asthma children. CASC2 may be involved in childhood asthma through inhibiting ASMCs proliferation, migration and inflammation via sponging miR-31-5p.

Keywords: asthma, lncRNA CASC2, miR-31-5p, ASMCs

\section{Introduction}

Asthma is a heterogeneous disease characterized by chronic airway inflammation and airway hyperresponsiveness. The clinical manifestations were heterogeneous and different degrees of airway inflammation and airway remodeling. ${ }^{1}$ It is the most common chronic respiratory disease in childhood because it is inherited and often occurs in early childhood, with serious impacts on children's health. ${ }^{2}$ Asthma is a chronic inflammatory disease of the airway that is co-acted by a variety of cells and their components, but its etiology is complex, and the specific pathogenesis is not fully understood. It has been reported that Airway remodeling starts early in childhood asthma. ${ }^{3,4}$ Airway remodeling is a proposed mechanism that underlies the persistent loss of lung function associated with childhood asthma. ${ }^{5}$ Airway smooth muscle cells (ASMCs) play an important role in airway remodeling. ${ }^{6}$ Abnormal proliferation of ASMCs and airway inflammation has been identified as a hallmark of asthma, especially in fatal or severe asthma. ${ }^{7}$ Hence, it is critical to explore the underlying mechanisms that regulate the proliferation and migration of ASMCs, inhibiting the changes of ASMCs may 
prevent the development of asthma. Current treatment methods cannot completely prevent airway remodeling and acute attacks. It is urgent to find more effective biomarkers to guide the diagnosis and treatment of asthma.

Long non-coding RNAs (lncRNAs) are regulatory non-coding RNAs with a length of more than 200 nucleotides and no protein coding function. LncRNAs are involved in regulating the physiological processes of various cells. Currently, studies on lncRNAs have focused on the occurrence and development of tumors. ${ }^{8}$ Recent findings proposed that lncRNAs serve as powerful regulators in immune response, and their expression profiles are different in patients with different types of asthma, suggesting that lncRNAs have a close relationship with the occurrence of asthma. ${ }^{9,10}$ Recent studies have found that lncRNA CASC2 is not only involved in the formation of a variety of malignant tumors, but also can regulate the inflammatory response of the body. ${ }^{11} \mathrm{CASC} 2$ is low expressed in lipopolysaccharide-induced acute lung injury cell model and is involved in regulating the release of inflammatory factors. ${ }^{11}$ CASC2 is poorly expressed in neonatal mice with bronchopulmonary dysplasia, and overexpression of CASC2 promotes the proliferation of bronchial epithelial cells, demonstrating its regulatory effect on neonatal lung injury. ${ }^{12}$ In addition, through bioinformatics analysis, Liao et al constructed an asthma-related competitive endogenous RNA (ceRNA) network and determined that CASC2 was a key asthma-related lncRNA. ${ }^{13}$ And the relationship between CASC2 and miR-31-5p has been reported. ${ }^{14}$ However, the expression pattern and function of CASC2 in asthma remain unclear.

The present study detected the level of CASC2 in the serum of asthmatic children, and further evaluated its clinical value and relationship with the inflammatory response in asthma. In the aspect of mechanism, based on the ceRNA theory, the CASC2-miR-31-5p ceRNA network was explored in platelet-derived growth factor-BB (PDGF-BB) treated ASMCs cell models.

\section{Materials and Methods}

\section{Clinical Data and Sample Collection}

A total of 70 patients with asthma admitted to the Second Affiliated Hospital of $\mathrm{Xi}^{6}$ an Medical University from August 2019 to January 2021 were selected as the research subjects, including 36 males and 34 females. All patients were diagnosed according to the Guidelines for the Diagnosis and Prevention of Childhood Bronchial Asthma, formulated by the Respiratory Group of the Chinese Medical Association in 2008. A parent or legal guardian of the children provided written informed consent. Exclusion criteria for patients were as follows: (1) patients with allergic rhinitis history; (2) Patients with a family history of asthma; (3) patients with psoriasis, systemic lupus erythematosus and other autoimmune diseases; (4) patients recently treated with glucocorticoids; (5) Patients with abnormal lung function detection. 66 healthy children who underwent physical examination in the same period were selected as the control group. This study was conducted under the supervision and approval of the Second Affiliated Hospital of Xi'an Medical University Ethics Committee in accordance with the Declaration of Helsinki.

$5 \mathrm{~mL}$ of fasting venous blood was taken from each subject. After centrifugation, the supernatant was taken and stored at $-20^{\circ} \mathrm{C}$ for inspection.

\section{Cell Culture and Treatment}

Human ASMCs were obtained from the American Type Culture Collection (ATCC), conventionally cultured in Vascular Cell Basal Medium containing Vascular Smooth Muscle Cell Growth Kit. Cells were incubated at $37{ }^{\circ} \mathrm{C}$ in an incubator containing 5\% CO2 and saturated humidity. The medium was changed every other day, and the cells were passed every 2 to 4 days.

Cells in logarithmic growth phase were selected and seeded into 96-well plates. When the adherent cells grew to a density of about $50-60 \%$, they were starved in serum-free medium for 24 hours and then underwent different treatment. To simulate the airway hyperresponsiveness model, cells were treated with $25 \mathrm{ng} / \mathrm{mL}$ of PDGF-BB for 24 hours. ${ }^{15}$ To regulate the levels of lncRNA CASC2 and miR-31-5p in ASMCs, cells were transfected with pcDNA-CASC2 or negative empty vector, the small-interfering RNA against CASC2 (si-CASC2: 5'-AGACUAUAAUGAUACCUUGGG -3';) or its negative control (si-NC: 5'-UUCUCCGAACGUGUCACGUUU-3';), miR-31-5p mimic (5'AGGCAAGAUGCUGGCAUAGCU-3') or inhibitor (5'-AGCUAUGCCAGCAUCUUGCCU) or their negative control 
(miR-NC: 5'-CAGUACUUUUGUGUAGUACAAA-3') using Lipofectamine 3000. The sequences were synthesized and provided by Shanghai Sangon Ltd. 48 hours after transfection, the cells were collected for subsequent cell experiments.

\section{qRT-PCR}

Total RNA was extracted from serum samples or cells by Trizol method, and cDNA was obtained by reverse transcription after qualified detection. Levels of CASC2 and miR-31-5p were measured through real-time quantitative polymerase chain reaction (qRT-PCR). Using GAPDH and U6 as internal reference genes respectively, the expression levels of CASC2 and miR-31-5p were calculated according to $2^{-\Delta \Delta \mathrm{Ct}}$ method. The primer sequences were as follows: CASC2 primers forward: 5'-GCACATTGGACGGTGTTTCC-3', reverse:5'- CCC AGTCCTTCACAGGTCAC-3'; GAPDH primers forward: 5'-CGCTCTC TGCTCCTCCTGTTC-3', reverse: 5'-ATCCGTTGACTC CGACCTTCAC3'; miR-31-5p primers forward: 5'-ACACTCCAGCTGGGAGGCAAGATGCTGGC-3', reverse: 5'TGGTGTCGTGGAGTCG-3'; U6 primers forward: 5'-CTCGCTTCGGCAGCACA-3' and reverse: 5'ACGCTTCACGAATTTGCGT-3'.

\section{CCK-8 Assay}

After digestion with $0.25 \%$ trypsin EDTA, $2 \times 10^{3}$ cells were collected and plated into 96 -well plate. Cells were cultured in an incubator at $37^{\circ} \mathrm{C}$ with $5 \% \mathrm{CO}_{2}$, and when the cells grew to $60-70 \%$ confluence, $25 \mathrm{ng} / \mathrm{mL}$ of PDGF-BB was added into the culture medium. After culturing for $24 \mathrm{~h}, 10 \mu \mathrm{L}$ CCK-8 reagent was added to each well, and placed in the incubator for further culture for $1 \mathrm{~h}$. After that, OD value of each well was measured with a microplate analyzer at $450 \mathrm{~nm}$.

\section{Transwell Assay}

Transwell was used for the measurement of migrated cells. $2 \times 10^{3}$ cells were collected and seeded into the upper chamber with a diameter of $8 \mu \mathrm{m}$. Then the lower chamber of Transwell was filled with $600 \mu \mathrm{L}$ medium containing 10\% FBS. After the cells were incubated for 24 hours, the chamber was taken out, and the medium in the hole was discarded. The un-migrated cells on the upper chamber were gently wiped with cotton swabs, and the migrated cells attached to the lower chamber were fixed with $4 \%$ paraformaldehyde for $30 \mathrm{~min}$, and the chamber was properly air-dried. After staining with crystal violet for $20 \mathrm{~min}$, the cell number was counted under an inverted phase-contrast microscope, and 5 fields were randomly selected to calculate the average amount.

\section{Enzyme-Linked Immunosorbent Assay (ELISA)}

The levels of human inflammatory cytokines, including interferon- $\gamma$ (IFN- $\gamma$ ), interleukin-4 (IL-4), IL-10, were detected using the human ELISA kit based on the manufacturer's requirements.

\section{Luciferase Activity Assay}

The binding site of CASC2 and miR-31-5p was predicted according to the bioinformatics website lncBase Predicted V.2, and the binding site sequence of CASC2 containing the miR-31-5p binding site was inserted into the luciferase reporter plasmid vector to construct wild-type plasmid (WT-CASC2). At the same time, the binding site of CASC2 and miR-31$5 \mathrm{p}$ was mutated by gene mutation technology and inserted into the reporter plasmid to construct a mutant plasmid (MUTCASC2). WT-CASC2 or MUT-CASC2 were co-transfected with miR-31-5p mimic or inhibitor into ASMCs, respectively. 48 hours after transfection, the luciferase activity was measured by a fluorescence spectrometer. The experiment was repeated three times, and the luciferase activity value of each group was calculated as the ratio of luciferase value of firefly to renilla luciferase values.

\section{Statistics Process}

SPSS 18.0 and GraphPad 7.0 were used for statistical analysis. Measurement data were expressed as mean \pm standard deviation (SD), and differences between groups were analyzed by ANOVA. Categorical variables were expressed as percentages, and $\chi^{2}$ test was used for comparison between groups. Pearson's correlation method was used to analyze the 
Table I Basic Clinical Information of the Participants

\begin{tabular}{|c|c|c|c|}
\hline Parameter & Healthy Individuals $(n=66)$ & Asthma Patients $(n=70)$ & $P$-value \\
\hline Gender (female/male) & $37 / 29$ & $34 / 36$ & 0.382 \\
\hline Age (years) & $7.17 \pm 1.82$ & $6.74 \pm 1.70$ & 0.163 \\
\hline BMI $\left(\mathrm{kg} / \mathrm{m}^{2}\right)$ & $24.21 \pm 3.01$ & $23.60 \pm 2.78$ & 0.225 \\
\hline $\mathrm{FEV}_{\mathrm{I}}(\%)$ & $114.19 \pm 12.82$ & $82.41 \pm 12.12$ & $<0.001$ \\
\hline $\lg E(K U / L)$ & $34.37 \pm 9.09$ & $|46.9| \pm 32.95$ & $<0.001$ \\
\hline $\mathrm{IL}-4(\mathrm{ng} / \mu \mathrm{L})$ & $391.35 \pm 71.94$ & $500.35 \pm 71.16$ & $<0.001$ \\
\hline IL-IO (ng/ $\mu \mathrm{L})$ & $229.14 \pm 39.42$ & $320.85 \pm 28.52$ & $<0.001$ \\
\hline $\mathrm{IFN}-\gamma(\mathrm{ng} / \mu \mathrm{L})$ & $257.46 \pm 68.25$ & $|88.75 \pm 5| .6 \mid$ & $<0.001$ \\
\hline $\mathrm{FeNO}(\mathrm{ppb})$ & $17.79 \pm 4.30$ & $34.94 \pm 9.00$ & $<0.001$ \\
\hline
\end{tabular}

Abbreviations: BMI, body mass index; FEVI, forced expiratory volume in one second; IgE, immunoglobulin E; IFN- $\gamma$, interferon- $\gamma$; IL-4, interleukin- I2; IL-4, interleukin-I0.

correlation of relevant indexes. Receiver operating characteristic (ROC) curves were drawn by univariate regression model to evaluate diagnostic sensitivity and specificity. A $P<0.05$ was defined as statistically significant.

\section{Results}

\section{General Data}

As shown in Table 1, the general data of the study subjects in different groups were recorded. 70 asthma children were recruited, aged from 4-10 years old, with a mean age of 6.74 \pm 1.70 , including 34 females and 36 males. In addition, 66 age and gender-matched healthy controls were recruited, including 37 females and 29 males. There was no significant difference in the body mass index (BMI) level between the two groups $(P>0.05)$. It was observed that in comparison with the control groups, patients with asthma had elevated immunoglobulin E (IgE), IL-4, IL-10, FeNO levels, and the difference reached a significant level $(P<0.05)$. Low levels of forced expiratory volume in one second (FEV1\%) and IFN- $\gamma$ were detected in children with asthma $(P<0.05)$.

\section{Levels of IncRNA CASC2 in Asthma Children}

Levels of CASC2 in the clinical serum samples were measured using qRT-PCR, and the difference between groups was tested using Student's $t$-test. CASC2 was identified in low expression in children with asthma, and the difference reached a significant level (Figure 1, P $<0.001$ ).

\section{Evaluation of the Clinical Values of CASC2 in Asthma}

Based on the serum CASC2 levels in healthy controls and asthma children, a ROC curve was established. It was calculated that the area under the curve (AUC) was 0.907 , with diagnostic sensitivity and specificity of $81.4 \%$ and $84.8 \%$ (Figure 2). The results demonstrated the diagnostic potential for the asthma.

In view of the abnormal expression of CASC2 in asthmatic children, the correlation between CASC2 and clinical indicators of asthmatic patients was further analyzed (Table 2). Pearson's correlation analysis results showed that serum CASC2 was significantly positively correlated with FEV1 $(\mathrm{r}=0.781, P<0.001)$ and IFN- $\gamma(\mathrm{r}=0.635, P<0.001)$ levels in children with asthma. Nevertheless, a remarkable negative correlation was detected for serum CASC2 with the levels of IgE (r $=-0.727, P<0.001)$, IL-4 $(\mathrm{r}=-0.766, P<0.001)$. IL-10 $(\mathrm{r}=-0.797, P<0.001)$ and FeNO $(\mathrm{r}=-0.777, P<$ 0.001). Furthermore, after the multiple linear regression analysis, the significant correlations between serum CASC2 and other indicators still existed (all $P<0.01$ ).

\section{Effects of CASC2 on Proliferation, Migration and Inflammatory Response of ASMCs}

Cell transfection was performed for cell transfection. As shown in Figure 3A, levels of CASC2 were increased by pcDNA3.1-CASC2 transfection, which was downregulated by si-CASC2 (Figure 3A). Overexpression of CASC2 inhibited the ASMCs proliferation and migration, but CASC2 knockdown had the opposite effect (Figure 3B and C). Besides, the release of Th1-related cytokine (IFN- $\gamma$ ) was further promoted (Figure 3D), whereas Th2 related cytokines 


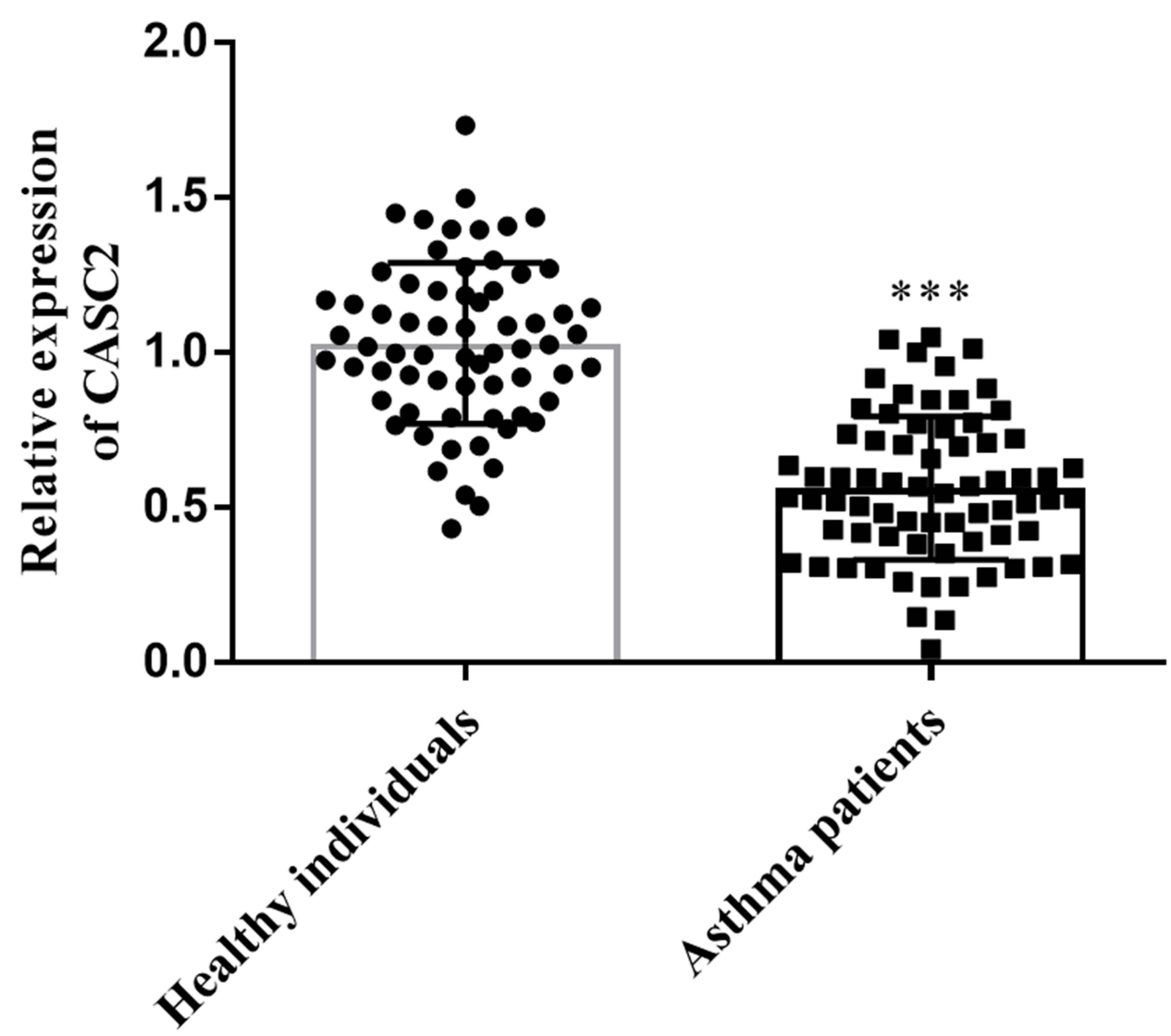

Figure I Levels of IncRNA CASC2 in the serum samples from healthy group and asthmatic patients' group. ***P $<0.00$ I when compared with healthy individuals' group. Abbreviation: CASC2, cancer susceptibility candidate 2 .

(IL-10 and IL-4) was suppressed by CASC2 upregulation (Figure 3E and F). CASC2 upregulation also elevated the mRNA levels of $\alpha$-SMA (Figure 3G).

Then the in-vitro asthma models were established using PDGF-BB, and the expression level of CASC2 in ASMCs was regulated by transfection of pcDNA-CASC2 and si-CASC2 (Figure 4A). Decreased level of CASC2 was detected in PDGFBB treated cell models, and the levels were reversely elevated by pcDNA-CASC2 transfection, but were significantly reduced by si-CASC2 transfection (Figure 4A). From the analysis results, CASC2 up-regulation inhibited PDGF-BB induced cell proliferation and migration, but the cell viability and migration were promoted by CASC 2 knockdown (Figure 4B and C, $\mathrm{P}<0.001$ ). In terms of inflammation, overexpression of CASC2 promoted the release of Th1-related cytokine (IFN- $\gamma$ ) (Figure 4D), but inhibited the release of Th2 related cytokines (IL-10 and IL-4, Figure 4E and F). Overexpression of CASC2 also elevated the mRNA level of $\alpha$-SMA which was reduced by PDGF-BB (Figure 4G). However, CASC2 knockout has the opposite effect on the release of IFN- $\gamma$, IL-10, IL-4 and $\alpha$-SMA (Figure 4D-G).

\section{LncRNA CASC2 Serves as a ceRNA of miR-3I-5p}

Through bioinformatics analysis, it was found that there was a direct targeting relationship between CASC2 and miR-31$5 \mathrm{p}$ (Figure 5A). The luciferase reporter assay results illustrated that in cells with WT-CASC2, miR-31-5p mimic transfection decreased the luciferase activity of cells, whereas miR-31-5p inhibitor transfection enhanced the luciferase activity (Figure 5B). Increased levels of miR-31-5p were measured in both in vitro cell models and asthmatic clinical serum samples (Figure 5C and D). Clinically, serum CASC2 showed a negative correlation with miR-31-5p levels in asthma children (Figure 5E, $\mathrm{r}=-0.864$ ). 


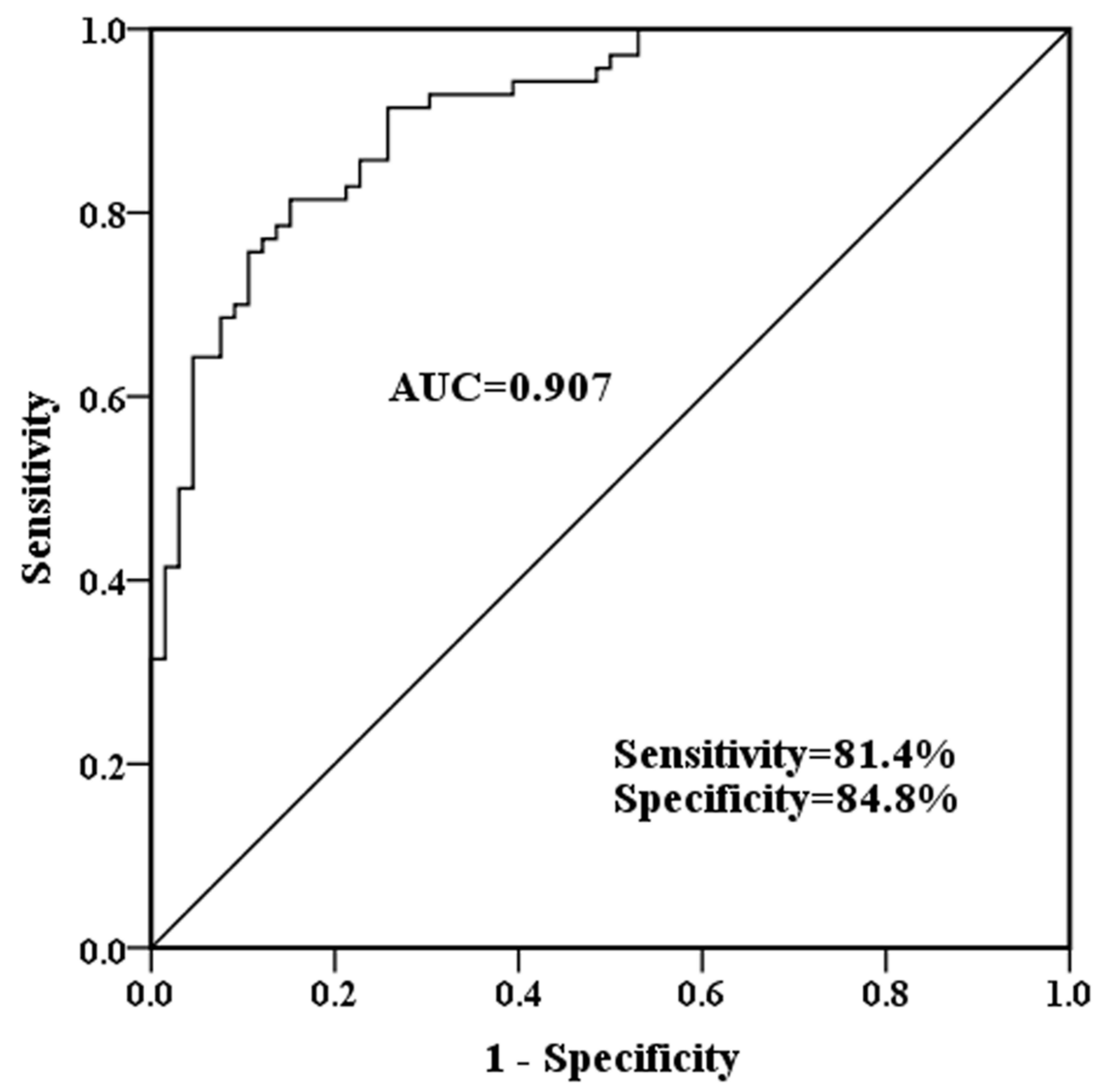

Figure 2 Serum CASC2 diagnostic value. ROC curve was made based on serum CASC2 levels in healthy controls and asthmatic children. Abbreviation: AUC, area under the curve.

\section{MiR-3I-5p Reversed the Influence of CASC2 in vitro}

To evaluate the role of miR-31-5p in asthma, miR-31-5p mimic was transfected into ASMCs using Lipofectamine 3000. The transfection efficiency was confirmed according to the qRT-PCR results. The results of Figure 6A showed that after miR-31-

Table 2 Correlation Between IncRNA CASC2 and Clinical Characteristics

\begin{tabular}{|c|c|c|c|c|c|c|}
\hline \multirow[t]{2}{*}{ Characteristics } & \multicolumn{2}{|c|}{ Bivariate Correlation } & \multicolumn{4}{|c|}{ Multiple Linear Regression } \\
\hline & Pearson(r) & $P$-value & Coefficient & $\begin{array}{c}\text { Standard } \\
\text { error }\end{array}$ & $\mathbf{t}$ & $P$-value \\
\hline $\mathrm{FEV}_{\mathrm{I}}(\%)$ & 0.781 & $<0.001$ & 0.004 & 0.001 & 4.340 & $<0.001$ \\
\hline $\lg E(K U / L)$ & -0.727 & $<0.001$ & 0.001 & 0.000 & 3.4668 & 0.001 \\
\hline 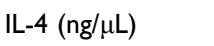 & -0.766 & $<0.001$ & -0.001 & 0.000 & -4.827 & $<0.001$ \\
\hline IL-I0 (ng/ $\mu \mathrm{L})$ & -0.797 & $<0.001$ & -0.001 & 0.000 & -4.035 & $<0.001$ \\
\hline $\mathrm{IFN}-\gamma(\mathrm{ng} / \mu \mathrm{L})$ & 0.635 & $<0.001$ & 0.001 & 0.000 & 2.814 & 0.006 \\
\hline FeNO (bbp) & -0.777 & $<0.001$ & -0.006 & 0.002 & -3.802 & $<0.001$ \\
\hline MiR-3I-5p & -0.864 & $<0.001$ & -0.277 & 0.051 & -5.423 & $<0.001$ \\
\hline
\end{tabular}

Abbreviations: BMI, body mass index; FEVI, forced expiratory volume in one second; IgE, immunoglobulin E; IFN- $\gamma$, interferon- $\gamma$; IL-4, interleukin-4; IL-I0, interleukin- I0. 


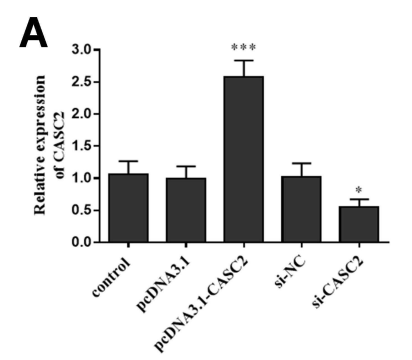

D

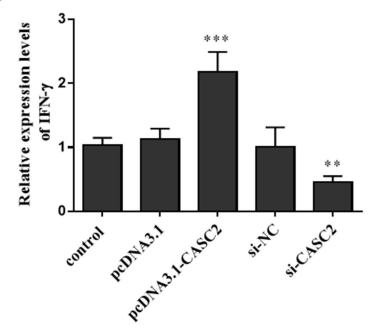

B

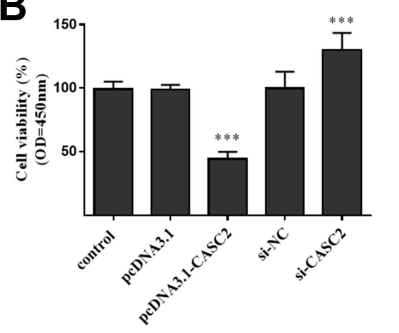

E

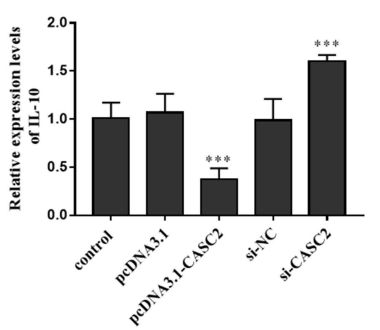

C
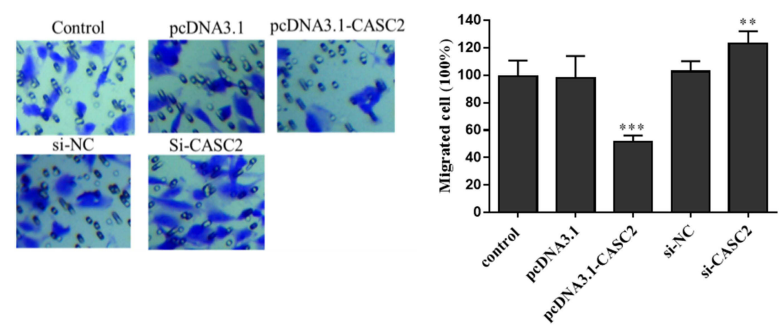

F

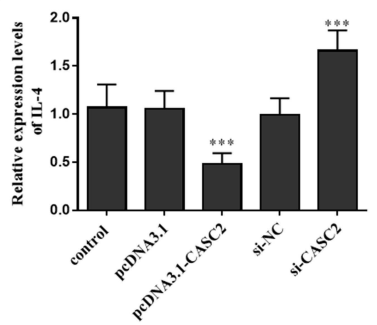

G

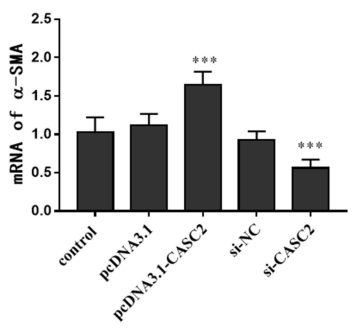

Figure 3 Effects of CASC2 on proliferation, migration and ThI/Th2 related cytokines of ASMCs. (A) The expression level of CASC2 in ASMCs was regulated by transfection of pcDNA-CASC2 and si-CASC2. (B) CASC2 up-regulation inhibited PDGF-BB induced cell proliferation, while the influence of CASC2 knockdown was opposite. (C) Role of CASC2 on ASMCs migration. (D) Effect of CASC2 expression on Th1 related cytokine (IFN- $\gamma$ ). (E and F) Effect of CASC2 expression on Th2 related cytokines (IL-10 and IL-4). (G) Levels of contractile phenotype marker ( $\alpha$-SMA) of ASMCs in different cell groups. **P $<0.01$, ***P $<0.001$ when compared with control group.

Abbreviations: CASC2, cancer susceptibility candidate 2; ASMC, airway smooth muscle cell; PDGF-BB, platelet-derived growth factors BB; IFN- $\gamma$, interferon- $\gamma$; IL-I0, interleukin-10; IL-4, interleukin-4; $\alpha$-SMA, $\alpha$-smooth muscle action; si-NC, negative control of the interfering RNA against CASC2; mRNA, message RNA.

A
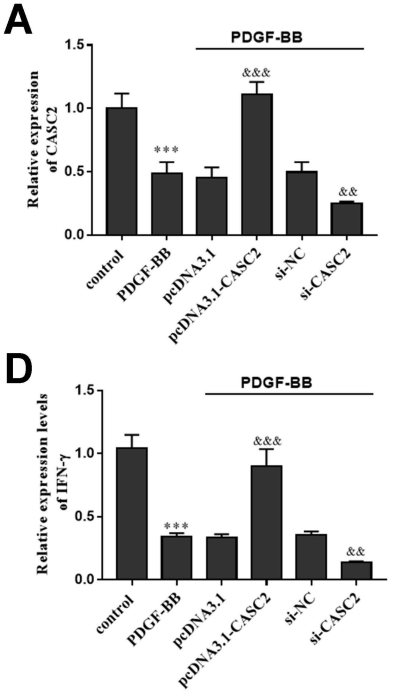

B

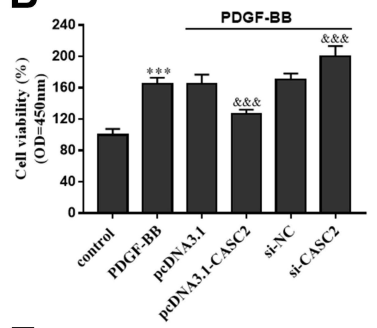

E

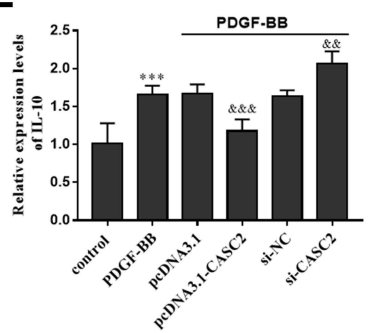

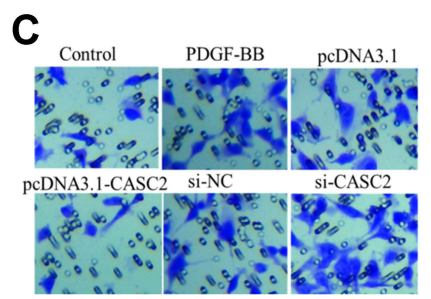

$\mathbf{F}$

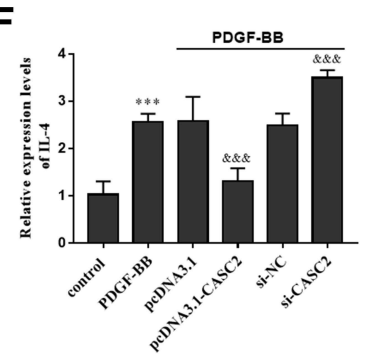

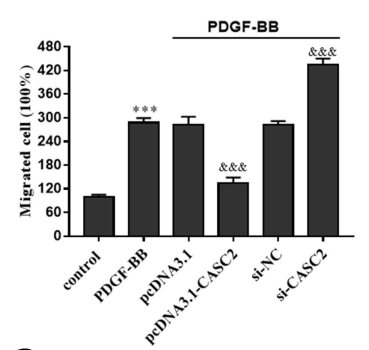

G

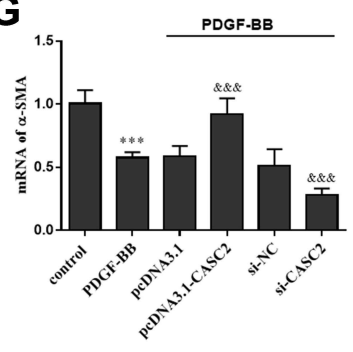

Figure 4 Role CASC2 in asthma ASMCs models induced by PDGF-BB. (A) Downregulation of CASC2 in ASMCs models and the cell transfection efficiency of pcDNACASC2 and si-CASC2. (B) Influence of CASC2 on ASMCs viability. (C) Influence of CASC2 on ASMCs migration. (D) Effect of CASC2 expression on Th1 related cytokine $(\mathrm{IFN}-\gamma)$. (E and $\mathbf{F}$ ) Effect of CASC2 expression on Th2 related cytokines (IL-10 and IL-4). (G) Influence of CASC2 on the mRNA levels of $\alpha-S M A$. ***P < $0.00 \mathrm{I}$ when compared with control group; ${ }^{\& \&} P<0.01$, ${ }^{\& \& \&} P<0.001$ when compared with PDGF-BB group.

Abbreviations: CASC2, cancer susceptibility candidate-2; ASMC, airway smooth cell; PDGF-BB, platelet-derived growth factors BB; IFN- $\gamma$, interferon- $\gamma$; IL-I0, interleukinI0; IL-4, interleukin-4; $\alpha$-SMA, $\alpha$-smooth muscle action; si-NC, negative control of the interfering RNA against CASC2; mRNA, message RNA.

$5 \mathrm{p}$ mimic transfection, miR-31-5p levels in ASMCs were raised $(P<0.001)$. The cell behaviors and inflammatory responses of cells under different treatments were detected. As CCK-8 and Transwell assay results reflected, overexpression of miR-31$5 \mathrm{p}$ significantly promoted ASMCs proliferation and migration, which reversed the effect of CASC 2 overexpression on cell proliferation and migration (Figure $6 \mathrm{~B}$ and $\mathrm{C}$ ). The influences on the release of inflammatory cytokines were further 


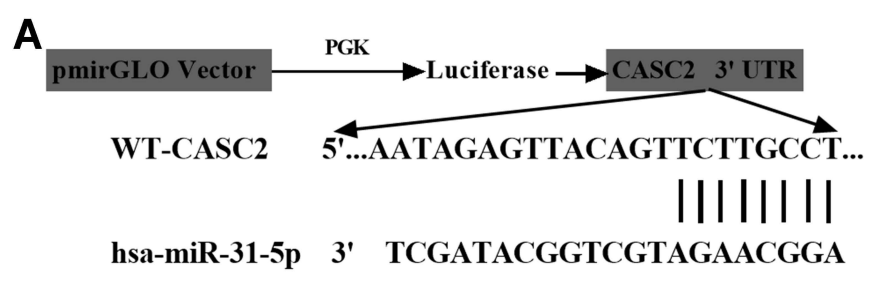

MUT-CASC2 5' AATAGAGTTACAGTTCAACGGA

C

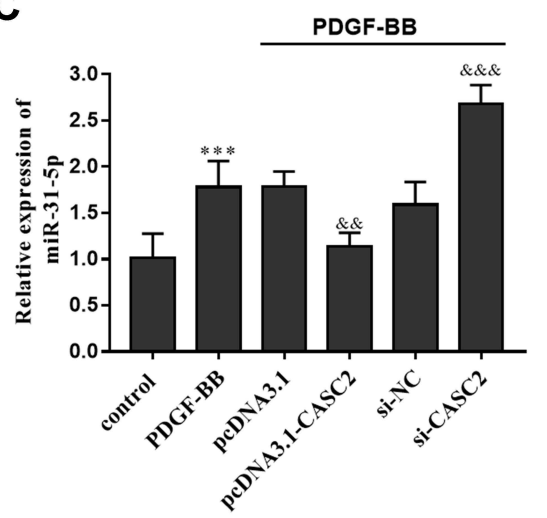

B

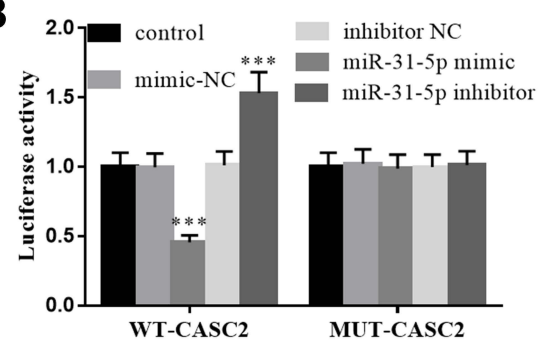

E
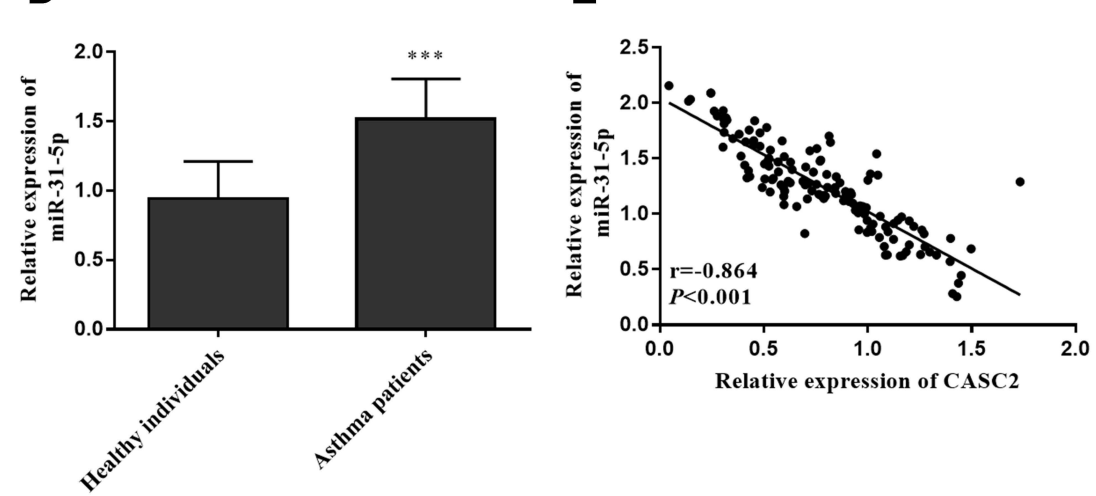

Figure 5 LncRNA CASC2 serves as a ceRNA of miR-3I-5p. (A) Targeting relationship between CASC2 and miR-3I-5p. (B) Luciferase activity of cells under different treatment. (C) Relative miR-3I-5p levels in different cell groups. (D) Serum miR-3I-5p levels in clinical serum samples. (E) Serum CASC2 showed a negative correlation with miR-3I-5p levels in all study subjects. $* * * P<0.001$ when compared with the control group; ${ }^{\& \&} P<0.01$, ${ }^{\& \&} P<0.00 \mathrm{I}$ when compared with PDGF-BB group.

Abbreviations: CASC2, cancer susceptibility candidate 2; NC, negative control; WT, wide type; MUT, mutant type; PDGF-BB, platelet-derived growth factors BB.

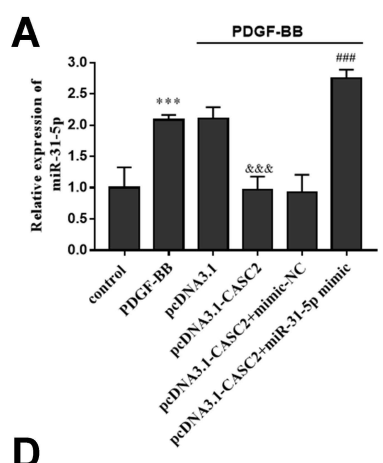

D

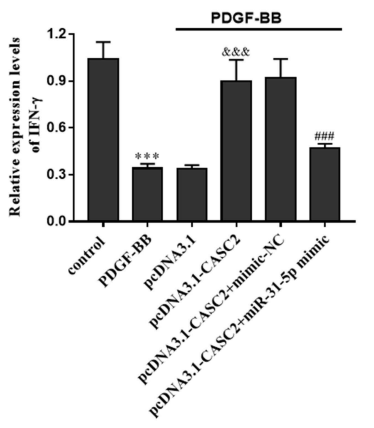

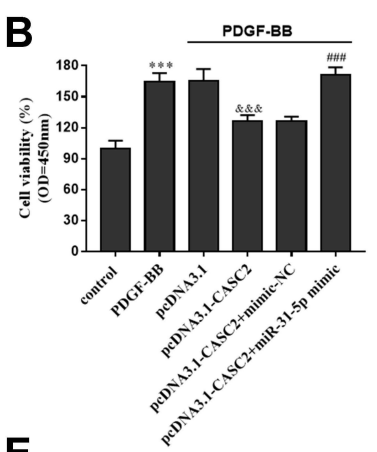

$\mathbf{E}$

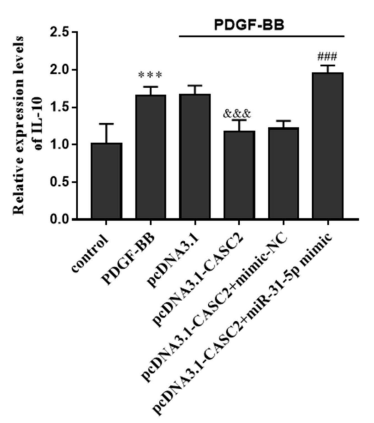

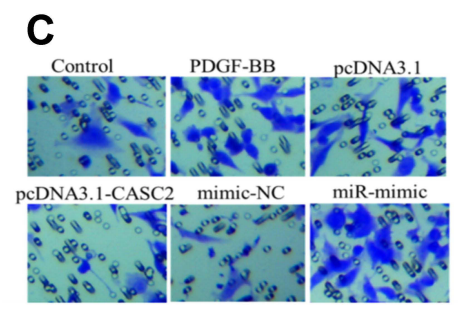

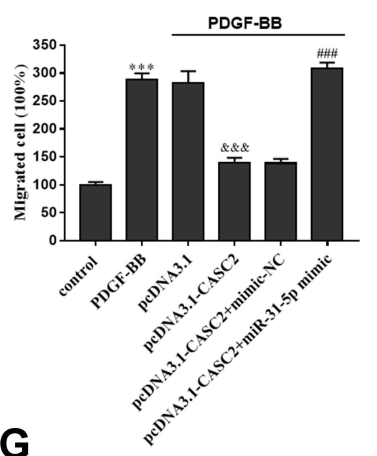

$\mathbf{F}$

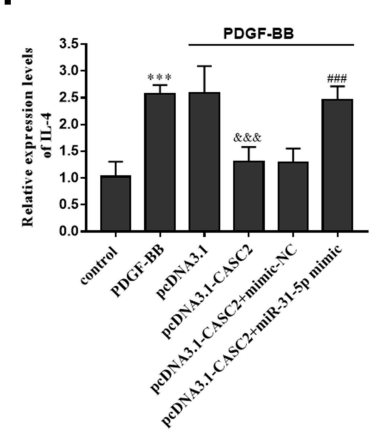

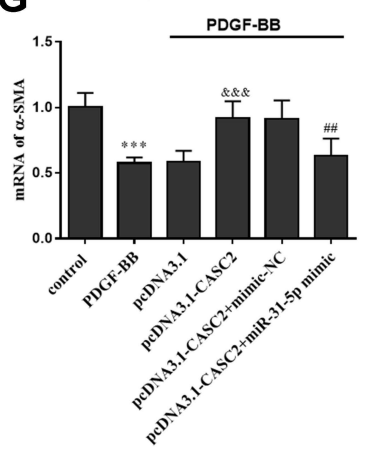

Figure 6 Overexpression of miR-3I-5p reversed the influence of CASC2 in vitro. (A) Relative miR-3I-5p levels in different cell groups. (B) Influence of miR-3I-5p on ASMCs proliferation. (C) Influence of miR-3I-5p on ASMCs migration. (D) Influence of miR-3I-5p on the release of ThI related cytokine (IFN- $\gamma$ ). (E and F) Influence of miR-3I-5p on the discharge of Th2 related cytokines (IL-10 and IL-4). (G) Influence of miR-3I-5p on the mRNA levels of $\alpha$-SMA. $* * * P<0.00 I$ when compared with control

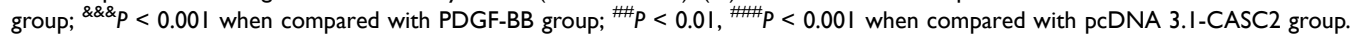

Abbreviations: CASC2, cancer susceptibility candidate 2; PDGF-BB, platelet-derived growth factors BB; NC, negative control; IFN- $\gamma$, interfeton- $\gamma$; IL-10, interleukin- I0; IL4, interleukin-4; $\alpha$-SMA, $\alpha$-smooth muscle action; mRNA, message RNA. 
explored. Based on ELISA results, overexpression of miR-31-5p counteracted the promotive effect on Th1 related factors (Figure 6D) and the inhibitory effect of CASC2 on Th2 related factors (Figure 6E and F). In addition, the promotion of $\alpha$ SMA induced by CASC2 was also reversed by miR-31-5p overexpression (Figure 6G).

\section{Discussion}

Asthma is a common chronic disease in children. In recent years, with the aggravation of environmental problems, its morbidity and mortality rates are gradually increasing, bringing a huge burden to public health security ${ }^{16}$ As previously reported, patients with asthma showed lower FEV1 and higher IgE levels during the acute episode. ${ }^{17,18}$ In the current study, 70 asthma children and 66 healthy controls were recruited. Consistent with the previous findings, lower FEV1 and higher IgE levels were detected in asthma children than in the healthy controls. It is speculated that it may be related to the dyspnea caused by asthma. In addition, Th2-related cytokines levels raised remarkably and the Th1 related factors decreased in asthma cases. The clinical data indicated that Th1/Th2 imbalance caused inflammatory reaction plays a critical influence in the asthma mechanism.

Asthma is a common chronic respiratory disease, which can occur in infancy and seriously affect children's health. If not treated promptly, it may lead to decreased lung function, and seriously affect the quality of life of children. ${ }^{19}$ It is of great significance to find the early diagnostic indicators of asthma, contributing to the effective control of the disease. LncRNAs can regulate the expression of related genes and thus participate in the occurrence of different diseases. ${ }^{20}$ Recent studies have shown that in addition to intracellular regulatory functions, IncRNAs and miRNAs can be secreted and detected in bodily fluids such as blood and urine. ${ }^{21}$ In agreement with the previous evidence, serum CASC2 and miR-31-5p were detected in asthma children according to the present findings. Abnormal expression of lncRNAs can also be used as biomarkers for early diagnosis of asthma, such as MEG3, ANRIL. ${ }^{22,23}$ The clinical data of this study showed that CASC2 was in low levels in the serum of asthma children, and correlated with the FEV1\% and IgE. It is suggested that CASC2 plays a regulatory role in the progression of asthma in children. Then the ROC curve analysis results demonstrated that serum CASC2 can serve as diagnostic biomarker for asthma. Several studies have reported the role of CASC2 in lung disease. In neonates with lung injury, overexpression of CASC2 can alleviate lung injury through inhibiting bronchial epithelial cell apoptosis. ${ }^{12}$ In lipopolysaccharids-induced acute lung injury models, CASC2 can inhibit the inflammatory response of A549 cells. ${ }^{11}$ These findings supported our hypothesis in terms of the potential role of CASC2 in asthma. However, the expression pattern of CASC2 at different stage of the childhood asthma was not analyzed, which is of great significance for future study.

The abnormal proliferation and migration of ASMCs are one of the pathogenesis of asthma airway remodeling. ${ }^{24}$ An increase in ASMCs could promote the airway obstruction and hyperresponsiveness. ASMCs migration may be the cause of airway remodeling and obstruction. ${ }^{25}$ In the absence of any stimulating factors, ASMCs cultured from asthmatic patients proliferate faster in vitro than that in non-asthmatic patients, especially in patients with fatal asthma, reflecting the crucial role of ASMCs in asthma. ${ }^{26}$ Platelet-derived growth factors BB (PDGF-BB) is a powerful mitotic growth factor, which can activate ASMCs and promote cell proliferation and migration under pathological conditions. ${ }^{27}$ PDGFBB-induced ASMC models have been widely used in the study of asthma. ${ }^{15}$ Excessive proliferation and migration of HASMCs directly accelerate the development of childhood asthma. In the current study, ASMCs were treated with $25 \mathrm{ng} /$ $\mathrm{mL}$ of PDGF-BB to mimic the asthma condition in vitro. Decreased level of CASC2 was detected in the cell models, the expression trend was consistent with that in the serum of clinical patients. The gain and loss function experiments uncovered that overexpression of CASC2 reversed the increased proliferation and migration of ASMCs induced by PDGF-BB, while CASC2 knockout further intensified the abnormal cell behavior. The phenotypic switch of vascular smooth muscle cells (VSMCs) is a key event in the pathogenesis of asthma. In PDGF-BB treated ASMCs, the reduced expression of contractile phenotype marker ( $\alpha$-SMA) was detected, but the trend was reversed by CASC 2 upregulation. The results supported our assumption in terms of the crucial role of CASC2 in asthma.

Airway inflammation is present at all stages of asthma and underlies clinical symptoms and airway hyperresponsiveness. ASMCs can secrete inflammatory factors, which further aggravate airway remodeling. ${ }^{28}$ The present in vitro experiments showed that PDGF-BB treated ASMCs can secrete more Th2 related cytokines, while the levels of Th1 related cytokines decreased, which was in accordance with the previous findings. Then the in vitro experiments 
prompted that elevating CASC2 inhibited the release of Th2 related cytokines, and elevated levels of Th1 related cytokines. CASC2 has been reported to exert anti-inflammatory effects in a variety of diseases. As Min et al reported, upregulation of CASC2 suppressed inflammatory factor release in high glucose (HG)-induced human renal mesangial cells, thus relieving the progression of diabetic nephropathy. ${ }^{29}$ In acute lung injury, CASC2 can inhibit the inflammatory response of cell models. ${ }^{11}$ In light of the anti-inflammatory action of CASC2 in asthma cell models, we deduced that CASC2 might be involved in the progression of asthma via regulating Th1/Th2 imbalance and inflammatory responses.

View from ceRNA theory, the action mechanism of CASC2 in asthma was further elucidated. Based on bioinformatics analysis and luciferase report assay results, miR-31-5p is proved to be the target gene of CASC2. Clinically, a negative association was also detected between serum CASC 2 and miR-31-5p levels in asthma patients. Consistently, elevated levels of miR-31-5p had been identified in both lung tissues and bronchoalveolar lavage fluid of asthma children and may mediate asthma progression via regulation inflammatory response. ${ }^{30}$ Then the present in vitro studies indicated that overexpression of miR-31-5p reversed the protective effect of CASC2 on ASMCs proliferation, migration, inflammatory factor release and $\alpha$-SMA. It can be envisioned that CASC2 may be involved in childhood asthma via sponging miR-31-5p. However, in this study, only cell experiments were carried out. In future studies, animal experiments are also meaningful to verify our present findings.

\section{Conclusion}

According to the present results, serum CASC2 is identified as a promising biomarker for the onset of asthma. CASC2 serves as a feasible positive regulator of ASMCs proliferation and migration and a negative mediator for cell inflammation. The present findings shed light on the interaction between CASC2 and miR-31-5p in asthma. In future studies, the underlying mechanisms that govern lncRNA CASC2-miR-31-5p interaction in asthma will be further excavated.

\section{Ethics Statement}

This study was conducted under the supervision and approval of the Second Affiliated Hospital of Xi 'an Medical University Ethics Committee. Written informed consent of each subject has been obtained.

\section{Disclosure}

The authors report no conflicts of interest in this work.

\section{References}

1. Chen Z, Liu NN, Xiao J, Wang YH, Dong R. The amygdala via the paraventricular nucleus regulates asthma attack in rats. CNS Neurosci Ther. 2020;26(7):730-740. doi:10.1111/cns.13293

2. Liu J, Liang R, Huang H, Zhang Y, Xie A, Zhong Y. Effect of an antagonistic peptide of CCR5 on the expression of autophagy-related genes and $\beta$ arrestin 2 in lung tissues of asthmatic mice. Allergy Asthma Immunol Res. 2021;13(1):106-121. doi:10.4168/aair.2021.13.1.106

3. Fleming L, Heaney L. Severe asthma-perspectives from adult and pediatric pulmonology. Front Pediatr. 2019;7:389. doi:10.3389/fped.2019.00389

4. Barbato A, Turato G, Baraldo S, et al. Epithelial damage and angiogenesis in the airways of children with asthma. Am J Respir Crit Care Med. 2006;174(9):975-981. doi:10.1164/rccm.200602-1890C

5. Reeves SR, Kolstad T, Lien TY, Herrington-Shaner S, Debley JS. Fibroblast-myofibroblast transition is differentially regulated by bronchial epithelial cells from asthmatic children. Respir Res. 2015;16:21. doi:10.1186/s12931-015-0185-7

6. Wang WL, Luo XM, Zhang Q, Zhu HQ, Chen GQ, Zhou Q. The lncRNA PVT1/miR-590-5p/FSTL1 axis modulates the proliferation and migration of airway smooth muscle cells in asthma. Autoimmunity. 2021;54(3):138-147. doi:10.1080/08916934.2021.1897977

7. Cheng SL. Immunologic pathophysiology and airway remodeling mechanism in severe asthma: focused on IgE-mediated pathways. Diagnostics. 2021;11(1):83.

8. Lu X, Wang F, Fu M, Li Y, Wang L. [ARTICLE WITHDRAWN] Long noncoding RNA KCNQ1OT1 accelerates the progression of ovarian cancer via microRNA-212-3/LCN2 axis. Oncol Res. 2020;28(2):135-146. doi:10.3727/096504019X15719983040135

9. Zhang XY, Zhang LX, Tian CJ, et al. LncRNAs BCYRN1 promoted the proliferation and migration of rat airway smooth muscle cells in asthma via upregulating the expression of transient receptor potential 1. Am J Transl Res. 2016;8(8):3409-3418.

10. Zhang J, Zhu Y, Wang R. Long noncoding RNAs in respiratory diseases. Histol Histopathol. 2018;33(8):747-756. doi:10.14670/HH-11-966

11. Li X, Mo J, Li J, Chen Y. IncRNA CASC2 inhibits lipopolysaccharideinduced acute lung injury via miR27b/TAB2 axis. Mol Med Rep. 2020;22 (6):5181-5190. doi:10.3892/mmr.2020.11606

12. Ji L, Liu Z, Dong C, Wu D, Yang S, Wu L. LncRNA CASC2 targets CAV1 by competitively binding with microRNA-194-5p to inhibit neonatal lung injury. Exp Mol Pathol. 2021;118:104575. doi:10.1016/j.yexmp.2020.104575 
13. Liao Y, Li P, Wang Y, Chen H, Ning S, Su D. Construction of asthma related competing endogenous RNA network revealed novel long non-coding RNAs and potential new drugs. Respir Res. 2020;21(1):14. doi:10.1186/s12931-019-1257-x

14. Wang J, Jia J, Zhou L. Long non-coding RNA CASC2 enhances cisplatin sensitivity in oral squamous cell cancer cells by the miR-31-5p/KANK1 axis. Neoplasma. 2021;67(6):1279-1292. doi:10.4149/neo 2020 191029N1102

15. Lin L, Li Q, Hao W, Zhang Y, Zhao L, Han W. Upregulation of lncRNA malat1 induced proliferation and migration of airway smooth muscle cells via miR-150-eIF4E/Akt signaling. Front Physiol. 2019;10:1337. doi:10.3389/fphys.2019.01337

16. Wang L, Zheng X, Hui Y, et al. Adjuvant treatment with Xiaoqinglong formula for bronchial asthma: protocol of systematic review and meta-analysis. Medicine. 2019;98(35):e17053. doi:10.1097/MD.0000000000017053

17. Lv H, Lu B, Qian XJ, Huang JA, Qiu TF. Serum IL-17 \& eotaxin levels in asthmatic patients with allergic rhinitis. Pak J Med Sci. 2016; 32 (3):700-704. doi:10.12669/pjms.323.9914

18. Abd El-Motaleb GS, Abou Amer AA, Elawa GM, Abo Alsood Abd Elfattah M. Study of urinary leukotriene E4 levels in children with acute asthma. Int J Gen Med. 2014;7:131-135. doi:10.2147/IJGM.S56660

19. de Sousa RB, Medeiros D, Sarinho E, Rizzo JA, Silva AR, Bianca AC. Risk factors for recurrent wheezing in infants: a case-control study. Rev Saude Publica. 2016;50:15. doi:10.1590/S1518-8787.2016050005100

20. Yu X, Zhe Z, Tang B, et al. $\alpha$-Asarone suppresses the proliferation and migration of ASMCs through targeting the lncRNA-PVT1/miR-203a/E2F3 signal pathway in RSV-infected rats. Acta Biochim Biophys Sin. 2017;49(7):598-608. doi:10.1093/abbs/gmx048

21. Hamdorf M, Kawakita S, Everly M. The potential of microRNAs as novel biomarkers for transplant rejection. J Immunol Res. 2017;2017:4072364. doi:10.1155/2017/4072364

22. Qiu YY, Wu Y, Lin MJ, Bian T, Xiao YL, Qin C. LncRNA-MEG3 functions as a competing endogenous RNA to regulate Treg/Th17 balance in patients with asthma by targeting microRNA-17/ROR $\gamma$ t. Biomed Pharmacother. 2019;111:386-394. doi:10.1016/j.biopha.2018.12.080

23. Ye S, Zhu S, Feng L. LncRNA ANRIL/miR-125a axis exhibits potential as a biomarker for disease exacerbation, severity, and inflammation in bronchial asthma. J Clin Lab Anal. 2020;34(3):e23092. doi:10.1002/jcla.23092

24. Li H, Yang T, Wu R, Chen T, Sun Z, Yang L. Salidroside inhibits platelet-derived growth factor-induced proliferation and migration of airway smooth muscle cells. $J$ Cell Biochem. 2019;120(4):6642-6650. doi:10.1002/jcb.27960

25. Salter B, Pray C, Radford K, Martin JG, Nair P. Regulation of human airway smooth muscle cell migration and relevance to asthma. Respir Res. 2017;18(1):156. doi:10.1186/s12931-017-0640-8

26. Wang Y, Zhang Y, Zhang M, et al. Saponins of dioscorea nipponicae inhibits IL-17A-induced changes in biomechanical behaviors of in vitro cultured human airway smooth muscle cells. J Eng Sci Med Diagn Ther. 2019;2(1):0110021-110027. doi:10.1115/1.4042317

27. Wang W, Nie W, Liu D, et al. Macroporous nanofibrous vascular scaffold with improved biodegradability and smooth muscle cells infiltration prepared by dual phase separation technique. Int J Nanomed. 2018;13:7003-7018. doi:10.2147/IJN.S183463

28. Pan LY, Han YQ, Wang YZ, Chen QQ, Wu Y, Sun Y. Mechanism of Yanghe Pingchuan granules treatment for airway remodeling in asthma. Drug Des Devel Ther. 2018;12:1941-1951. doi:10.2147/DDDT.S159428

29. Min XQ, Xie Y. LncRNA CASC2 alleviates the progression of diabetic nephropathy by regulating the miR-144/SOCS2 signalling axis. Kidney Blood Press Res. 2020;45(6):837-849. doi:10.1159/000508078

30. Shi ZG, Sun Y, Wang KS, Jia JD, Yang J, Li YN. Effects of miR-26a/miR-146a/miR-31 on airway inflammation of asthma mice and asthma children. Eur Rev Med Pharmacol Sci. 2019;23(12):5432-5440. doi:10.26355/eurrev_201906_18212

Journal of Asthma and Allergy

Dovepress

\section{Publish your work in this journal}

The Journal of Asthma and Allergy is an international, peer-reviewed open-access journal publishing original research, reports, editorials and commentaries on the following topics: Asthma; Pulmonary physiology; Asthma related clinical health; Clinical immunology and the immunological basis of disease; Pharmacological interventions and new therapies. The manuscript management system is completely online and includes a very quick and fair peer-review system, which is all easy to use. Visit http://www.dovepress.com/testimonials.php to read real quotes from published authors.

Submit your manuscript here: https://www.dovepress.com/journal-of-asthma-and-allergy-journal 